\title{
New Impossible Differential Attacks of Reduced-Round Camellia-192 and Camellia-256 ${ }^{\star}$
}

\author{
Jiazhe Chen ${ }^{1,2}$, Keting $\mathrm{Jia}^{3}$, Hongbo $\mathrm{Yu}^{4}$, and Xiaoyun Wang ${ }^{1,2,3 \star \star}$ \\ 1 Key Laboratory of Cryptologic Technology and Information Security, \\ Ministry of Education, Shandong University, Jinan 250100, China \\ 2 School of Mathematics, Shandong University, Jinan 250100, China \\ jiazhechen@mail.sdu.edu.cn \\ 3 Institute for Advanced Study, Tsinghua University, Beijing 100084, China \\ ${ }^{4}$ Department of Computer Science and Technology, Tsinghua University, Beijing 100084, China \\ \{ktjia, yuhongbo, xiaoyunwang\}@mail.tsinghua.edu.cn
}

\begin{abstract}
Camellia is a block cipher selected as a standard by ISO/IEC, which has been analyzed by a number of cryptanalysts. In this paper, we propose several 6-round impossible differential paths of Camellia with the $F L / F L^{-1}$ layer in the middle of them. With the impossible differential and a well-organized precomputational table, impossible differential attacks on 10-round Camellia-192 and 11-round Camellia-256 are given, and the time complexity are $2^{175}$ and $2^{206.8}$ respectively. An impossible differential attack on 15-round Camellia-256 without $F L / F L^{-1}$ layers and whitening is also be given, which needs about $2^{236.1}$ encryptions. To the best of our knowledge, these are the best cryptanalytic results of Camellia-192/-256 with $F L / F L^{-1}$ layers and Camellia-256 without $F L / F L^{-1}$ layers to date.
\end{abstract}

Key words: Camellia Block Cipher, Cryptanalysis, Impossible Differential Path, Impossible Differential Attack.

\section{Introduction}

Block cipher Camellia is proposed by NTT and Mitsubishi in 2000 [1]. Its block size is 128 bits and it supports 128-, 192- and 256-bit key sizes with 18, 24 and 24 rounds respectively. Camellia was selected as an e-government recommended cipher by CRYPTREC [5] and recommended in NESSIE [15] block cipher portfolio. Then it was selected as an international standard by ISO/IEC.

The structure of Camellia is Feistel structure with $F L / F L^{-1}$ layers inserted every 6 rounds. The $F L$ and $F L^{-1}$ functions are keyed linear functions which are designed to provide nonregularity across rounds [1]. In the past years, Camellia has attracted the attention of the cryptanalytic community. The square-type attacks are efficient to attack Camellia, which can be used to analysis 9-round Camellia-128 and 10-round Camellia-256 [11]. Furthermore, Hatano et al. used the higher order differential attack to analysis the last 11 rounds Camellia-256 with complexity $2^{255.6}[7]$.

There are a number of results on simple versions of Camellia which exclude the $F L / F L^{-1}$ layers and whitening being given in recent years [6]10]13]14]16]17,18]19]. Among them, the impossible differential attacks [3] are most efficient [13/14]17]18]. Since the existence of $F L / F L^{-1}$ layers will probably destroy the impossibility, non of the impossible differential paths in these attacks includes the $F L / F L^{-1}$ layers. In this paper, we present 6-round impossible differential paths with $F L / F L^{-1}$ layers in the middle, which turn out to be first impossible differential

\footnotetext{
* Supported by 973 Project (No.2007CB807902), the National Natural Science Foundation of China (Grant No.60931160442) and Graduate Independent Innovation Foundation of Shandong University (No. $11140070613183)$.

** Corresponding author
} 
paths with $F L / F L^{-1}$ layers. Due to one of these impossible differential paths and a precomputational table that is carefully consturcted, we propose impossible differential attacks on 10-round Camellia-192 and 11-round (Round 1-11) Camellia-256 with complexity $2^{175}$ and $2^{206.8}$ respectively.

For the attacks of Camellia-256 without $F L / F L^{-1}$ layers and whitening, the 14-round attack in [13] was pointed out to be incorrect by [20]. Later Mala et al. [14] pointed out a flaw in [20] and showed that the time complexities of the 12-round Camellia-128 and 16-round Camellia-256 attacks were more than exhaustive search. As a result, the best analysis of Camellia-256 without $F L / F L^{-1}$ layers and whitening dated back to [12], which was a 13-round attack with complexity $2^{211.7}$. By carefully using the subkey relations and one of the 8-round impossible differential paths without $F L / F L^{-1}$ layers proposed in [18], we also present an impossible differential attack on 15 -round Camellia-256 without $F L / F L^{-1}$ layers and whitening, and the complexity is about $2^{236.1}$ encryptions.

The rest of this paper is organized as follows. We give some notations and a brief description of Camellia in Section 2. Some properties and 6-round impossible differential paths with $F L / F L^{-1}$ layers of Camellia are given in Section 3 . Section 4 describes the impossible differential attacks on reduced-round Camellia with $F L / F L^{-1}$ layers and whitening. The impossible differential attack on 15-round Camellia-256 without $F L / F L^{-1}$ layers and whitening is illustrated in Section 5 . Finally, we conclude the paper in Section 6 .

\section{Preliminaries}

Some notions used in this paper and a simple description of the Camellia algorithm are given in this section.

\subsection{Notations}

$L^{r-1}, L^{\prime r-1}:$ the left half of the 128-bit $r$-th round input

$R^{r-1}, R^{\prime r-1}$ : the right half of the 128-bit $r$-th round input

$\Delta L^{r-1}$ : the difference of $L^{r-1}, L^{\prime r-1}$

$\Delta R^{r-1}$ : the difference of $R^{r-1}, R^{\prime r-1}$

$S^{r}, S^{\prime r}$ : the output value of the S-box of the $r$-th round

$\Delta S^{r}$ : the output difference of the S-box of the $r$-th round

$k^{r}$ : the 64 -bit $r$-th round subkey,

$A_{i}$ : the $i$-th byte of a 64 -bit value $A(i=1, \ldots, 8)$

$B \lll j$ : left rotation of $B$ by $j$ bits

$X_{L(64)}$ : the left half of a 128 -bit word $X$

$X_{R(64)}$ : the right half of a 128-bit word $X$

$Y_{L(32)}$ : the left half of a 64 -bit word $Y$

$Y_{R(32)}$ : the right half of a 64 -bit word $Y$

$\|$ : the cascade of two words

\subsection{The Camellia Algorithm}

Camellia [1] is a 128-bit block cipher with Feistel structure. It has 18 rounds for 128-bit key, and 24 rounds for 192-/256-bit key. We give the encryption procedure of Camellia-192/-256 as follows, see Fig. 1 .

Encryption Procedure. The input of the encryption procedure is a 128-bit plaintext $M$, and 64-bit subkeys $k^{w i}(i=1, \ldots, 4), k^{r}(r=1, \ldots, 24)$ and $k l^{j}(j=1, \ldots, 6)$. First $M$ is XORed with 


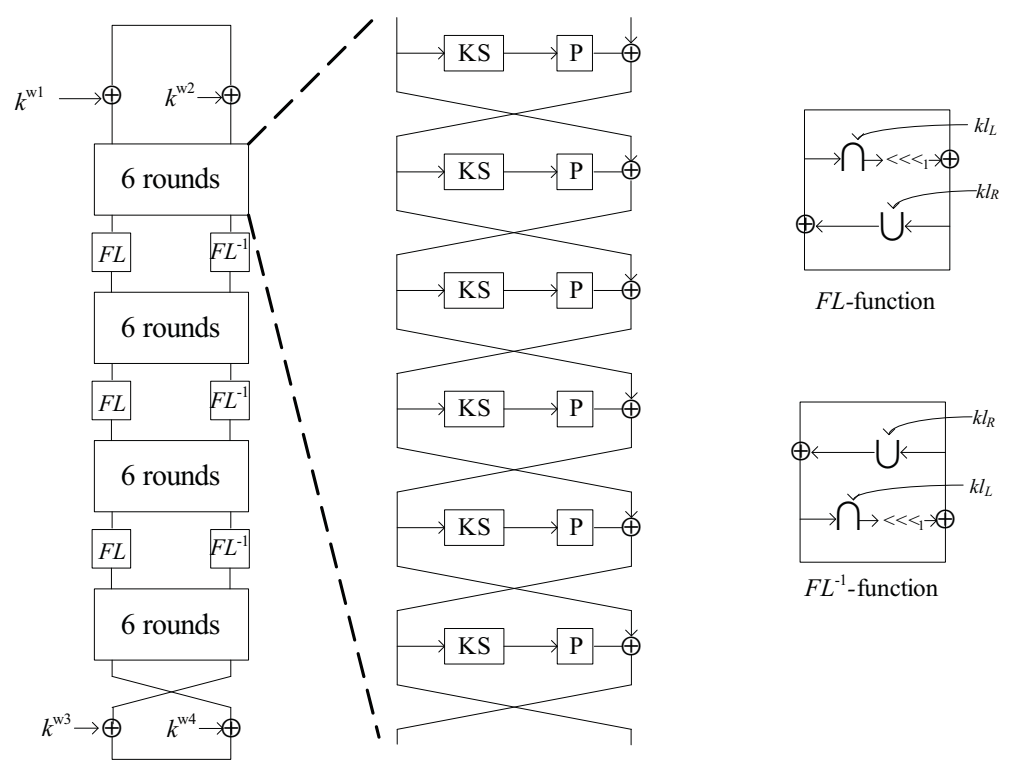

Fig. 1. Camellia-192/-256

$k^{w 1}$ and $k^{w 2}$ to get two 64 -bit intermediate value $L^{0}$ and $R^{0}: L^{0} \| R^{0}=M \oplus\left(k^{w 1} \| k^{w 2}\right)$. Then the following operations are carried out for $i=1$ to 24 , expect for $r=6,12$ and 18:

$$
L^{r}=R^{r-1} \oplus F\left(L^{r-1}, k^{r}\right), R^{r}=L^{r-1} .
$$

For $r=6,12$ and 18 , do the following:

$$
\begin{aligned}
L^{\prime r} & =R^{r-1} \oplus F\left(L^{r-1}, k^{r}\right), R^{\prime r}=L^{r-1} . \\
L^{r} & =F L\left(L^{\prime r}, k l^{2 r / 6-1}\right), R^{r}=F L^{-1}\left(R^{\prime r}, k l^{2 r / 6}\right) .
\end{aligned}
$$

Finally the 128-bit ciphertext $C$ is computed as: $C=\left(R^{24} \| L^{24}\right) \oplus\left(k^{w 3} \| k^{w 4}\right)$.

The $F L$ function is defined as: $\left(X_{L(32)}\left\|X_{R(32)}, k l_{L(32)}\right\| k l_{R(32)}\right) \mapsto\left(Y_{L(32)} \| Y_{R(32)}\right)$, where:

$$
\begin{aligned}
& Y_{R(32)}=\left(\left(X_{L(32)} \cap k l_{L(32)}\right) \lll 1\right) \oplus X_{R(32)}, \\
& Y_{L(32)}=\left(Y_{R(32)} \cup k l_{R(32)}\right) \oplus X_{L(32)} .
\end{aligned}
$$

The $F L^{-1}$ function is the inverse of $F L$ function, and $F L$ and $F L^{-1}$ are linear as long as the key is fixed [2].

The round function $F$ is composed of the key-addition layer, S-box layer and linear transformation $P$. In the key-addition layer, the input of the round function is XORed with the subkey. There are $48 \times 8$ S-boxes $S_{1}, S_{2}, S_{3}, S_{4}$ used in the S-box layer, and each S-box is used twice. Finally, the linear transformation $P:\left(\{0,1\}^{8}\right)^{8} \rightarrow\left(\{0,1\}^{8}\right)^{8}$ maps $\left(z_{1}, \ldots, z_{8}\right) \rightarrow\left(y_{1}, \ldots, y_{8}\right) . P$ function and its inverse function $P^{-1}$ are:

$$
\begin{aligned}
& y_{1}=z_{1} \oplus z_{3} \oplus z_{4} \oplus z_{6} \oplus z_{7} \oplus z_{8} \\
& y_{2}=z_{1} \oplus z_{2} \oplus z_{4} \oplus z_{5} \oplus z_{7} \oplus z_{8} \\
& y_{3}=z_{1} \oplus z_{2} \oplus z_{3} \oplus z_{5} \oplus z_{6} \oplus z_{8} \\
& y_{4}=z_{2} \oplus z_{3} \oplus z_{4} \oplus z_{5} \oplus z_{6} \oplus z_{7} \\
& y_{5}=z_{1} \oplus z_{2} \oplus z_{6} \oplus z_{7} \oplus z_{8} \\
& y_{6}=z_{2} \oplus z_{3} \oplus z_{5} \oplus z_{7} \oplus z_{8} \\
& y_{7}=z_{3} \oplus z_{4} \oplus z_{5} \oplus z_{6} \oplus z_{8} \\
& y_{8}=z_{1} \oplus z_{4} \oplus z_{5} \oplus z_{6} \oplus z_{7}
\end{aligned}
$$

$$
\begin{aligned}
& z_{1}=y_{2} \oplus y_{3} \oplus y_{4} \oplus y_{6} \oplus y_{7} \oplus y_{8} \\
& z_{2}=y_{1} \oplus y_{3} \oplus y_{4} \oplus y_{5} \oplus y_{7} \oplus y_{8} \\
& z_{3}=y_{1} \oplus y_{2} \oplus y_{4} \oplus y_{5} \oplus y_{6} \oplus y_{8} \\
& z_{4}=y_{1} \oplus y_{2} \oplus y_{3} \oplus y_{5} \oplus y_{6} \oplus y_{7} \\
& z_{5}=y_{1} \oplus y_{2} \oplus y_{5} \oplus y_{7} \oplus y_{8} \\
& z_{6}=y_{2} \oplus y_{3} \oplus y_{5} \oplus y_{6} \oplus y_{8} \\
& z_{7}=y_{3} \oplus y_{4} \oplus y_{5} \oplus y_{6} \oplus y_{7} \\
& z_{8}=y_{1} \oplus y_{4} \oplus y_{6} \oplus y_{7} \oplus y_{8}
\end{aligned}
$$



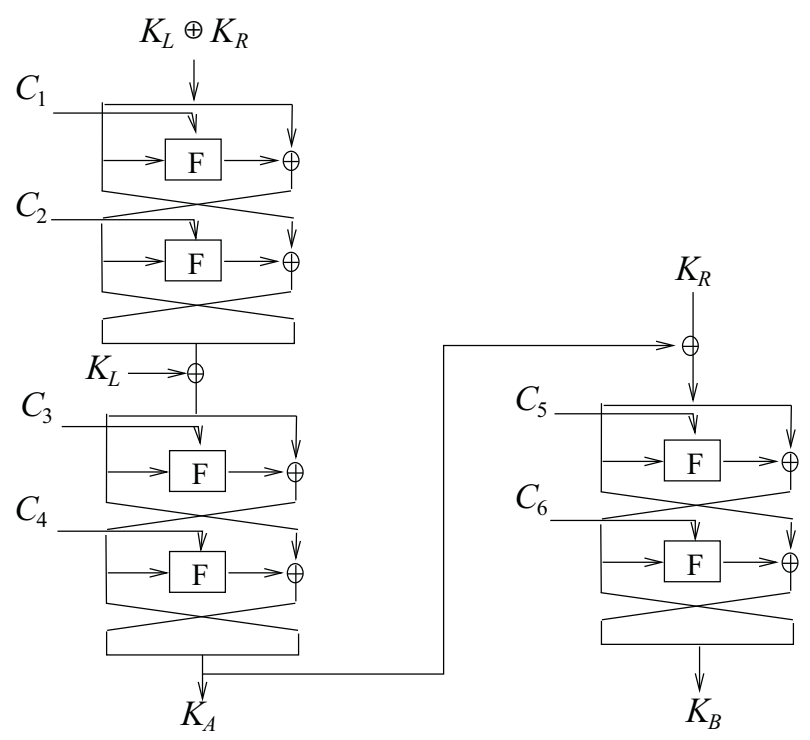

Fig. 2. The Calculation of $K_{A}$ and $K_{B}$

Key Schedule. For Camellia-256, the 256-bit main key $K=K_{L} \| K_{R}$, where $K_{L}$ and $K_{R}$ are 128 bits. And for Camellia-192, the 192-bit main key $K=K_{L} \| K_{R L(64)}$ and $K_{R R(64)}=\overline{K_{R L(64)}}$. Using $K_{L}$ and $K_{R}$, the key schedule algorithm first calculate $K_{A}$ and $K_{B}$, which is described in Fig. 2. Where $F$ is the round function of Camellia and $C_{i}(1 \leq i \leq 6)$ are constants used as the keys. Then the subkeys $k^{w i}(i=1, \ldots, 4), k^{r}(r=1, \ldots, 24)$ and $k l^{j}(j=1, \ldots, 6)$ are derived from rotating $K_{L}, K_{R}, K_{A}$ or $K_{B}$. For details of Camellia, we refer to [1].

It can be known from Fig. 2 that, if $K_{B}$ and $K_{R}$ are known, $K_{A}$ is known. Therefore, one can get $K_{L}$ using the relation between $K_{L}$ and $K_{A}$ described in [14, Section 3.2. So once $K_{B}$ and $K_{R}$ are known, $K$ can be computed.

\section{Properties and 6-Round Impossible Differential Paths of Camellia with $F L / F L^{-1}$ Functions}

In this section, we first give some useful properties of Camellia and then propose several impossible differential paths.

Property 1 For a 3-round Camellia structure, if the input difference is of the form $\Delta L^{i}=$ $(0, a, 0,0,0,0,0,0), \Delta R^{i}=(0,0,0,0,0,0,0,0)$, then:

$\Delta L^{i+1}=(0, b, b, b, b, b, 0,0), \Delta S^{i+2}=\left(0, b_{2}, b_{3}, b_{4}, b_{5}, b_{6}, 0,0\right)$,

$\Delta L^{i+2}=\Delta R^{i+3}=P\left(a, b_{2}, b_{3} \oplus a, b_{4} \oplus a, b_{5} \oplus a, b_{6} \oplus a, 0,0\right)$,

and $\Delta S_{l}^{i+3}=\left(P^{-1}\left(\Delta L^{i+3}\right)\right)_{l}$, for $l=1,3,4, \ldots, 8$, where $a, b, b_{2}, b_{3}, b_{4}, b_{5}, b_{6}$ are non-zero bytes.

Property 2 The necessary conditions of $\Delta L^{i+3}=(0, a, 0,0,0,0,0,0)$ and $\Delta R^{i+3}=(0,0,0,0,0$, $0,0,0)$ are:

$\Delta L^{i+1}=(0, b, b, b, b, b, 0,0), \Delta S^{i+2}=\left(0, b_{2}, b_{3}, b_{4}, b_{5}, b_{6}, 0,0\right)$,

$\Delta L^{i}=P\left(a, b_{2}, b_{3} \oplus a, b_{4} \oplus a, b_{5} \oplus a, b_{6} \oplus a, 0,0\right)$,

and $\Delta S_{l}^{i+1}=\left(P^{-1}\left(\Delta R^{i}\right)\right)_{l}$, for $l=1,3,4, \ldots, 8$, where $a, b, b_{2}, b_{3}, b_{4}, b_{5}, b_{6}$ are non-zero bytes.

To better describe the properties, we also illustrate them in Fig. 3. Actually, the proofs of the properties are similar and the proof Property 1 is given as an example. 


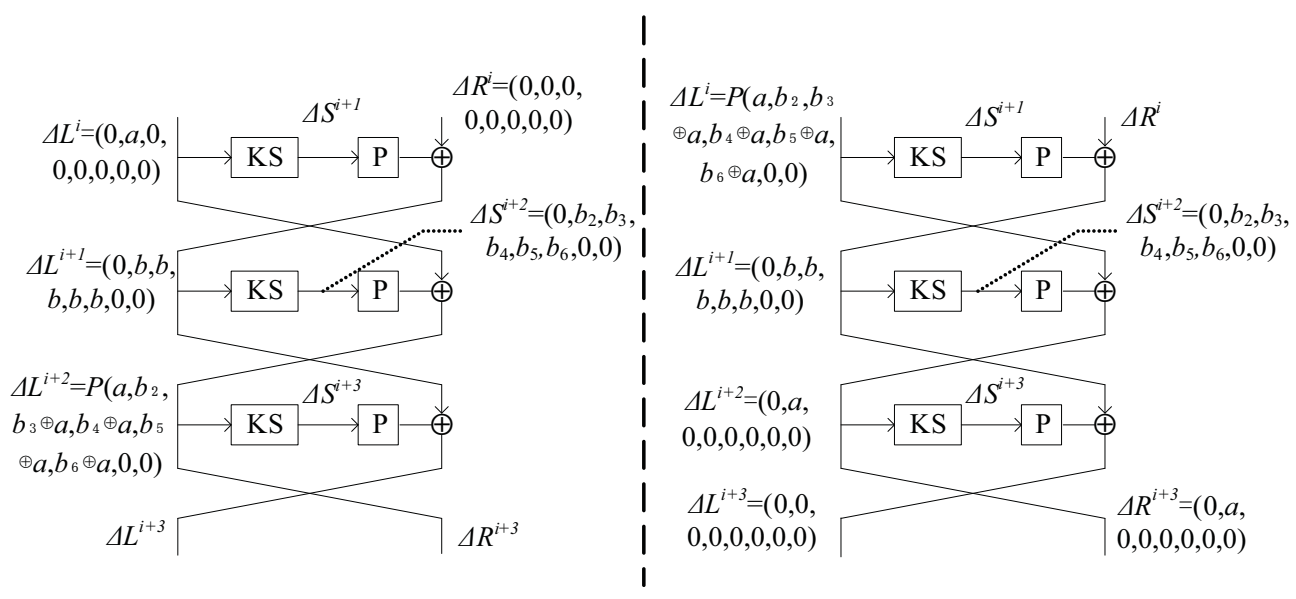

Fig. 3. Properties of 3-round Camellia

Proof. Apparently, $\Delta S^{i+1}$ is of the form $(0, b, 0,0,0,0,0,0)$, where $b$ an is unknown non-zero byte. And $\Delta L^{i+1}=(0, b, b, b, b, b, 0,0)$ as $P$ function is linear. After the key-addition layer and S-box layer, it can be obtained that $\Delta S^{i+2}=\left(0, b_{2}, b_{3}, b_{4}, b_{5}, b_{6}, 0,0\right)$, where $b_{2}, b_{3}, b_{4}, b_{5}$ and $b_{6}$ are unknown non-zero bytes.

Since $\Delta L^{i+2}=\Delta S^{i+2} \oplus \Delta L^{i}$ and $P^{-1}\left(\Delta L^{i}\right)=(a, 0, a, a, a, a, 0,0)$,

$$
\Delta L^{i+2}=P\left(a, b_{2}, b_{3} \oplus a, b_{4} \oplus a, b_{5} \oplus a, b_{6} \oplus a, 0,0\right) .
$$

Finally, because $\Delta S^{i+3}=P^{-1}\left(\Delta L^{i+1} \oplus \Delta L^{i+3}\right), P^{-1}\left(\Delta L^{i+1}\right)=(0, b, 0,0,0,0,0,0)$ and $P^{-1}$ function is linear,

$$
\Delta S_{l}^{i+3}=\left(P^{-1}\left(\Delta L^{i+3}\right)\right)_{l}, \text { for } l=1,3,4, \ldots, 8 .
$$

Property 3 (from [9]) Let $x, x^{*}$ be 32-bit values, and $x^{\prime}=x \oplus x^{*}$, then the differential properties of $A N D$ and $O R$ operations are:

$$
\begin{aligned}
& (x \cap k) \oplus\left(x^{*} \cap k\right)=\left(x \oplus x^{*}\right) \cap k=x^{\prime} \cap k \\
& (x \cup k) \oplus\left(x^{*} \cup k\right)=(x \oplus k \oplus(x \cap k)) \oplus\left(x^{*} \oplus k \oplus\left(x^{*} \cap k\right)\right)=x^{\prime} \oplus\left(x^{\prime} \cap k\right)
\end{aligned}
$$

Property 4 Let $M=\left(m_{1}, m_{2}, m_{3}, m_{4}, m_{5}, m_{6}, m_{7}, m_{8}\right)$ be the input difference of $F L$ function, and $N=\left(n_{1}, n_{2}, n_{3}, n_{4}, n_{5}, n_{6}, n_{7}, n_{8}\right)$ be the the output difference of $F L$, where $n_{l}, m_{l}(l=$ $1, \ldots, 8)$ are arbitrary 8-bit values. Then if $n_{i}=0(i \in\{5,6,7,8\}), n_{i-4}=m_{i-4}$.

Proof. Let us denote the subkey used for AND operation as $k_{L}$ and the subkey used for OR operation as $k_{R}$. By Property 3 , the following equations must hold:

$$
\begin{aligned}
\left(\left(M_{L} \cap k_{L}\right) \lll 1\right) \oplus M_{R} & =N_{R} \\
M_{L} \oplus N_{R} \oplus\left(N_{R} \cap k_{R}\right) & =N_{L}
\end{aligned}
$$

Then if $n_{i}=0(i \in\{5,6,7,8\})$, it can be deduced from Equation (1) that $n_{i-4}=m_{i-4}$.

Impossible Differential. Now we demonstrate that the 6-round differential in Fig. 4 is impossible. The input difference is

$$
((0,0,0,0,0,0,0,0) ;(0, a, 0,0,0,0,0,0))
$$




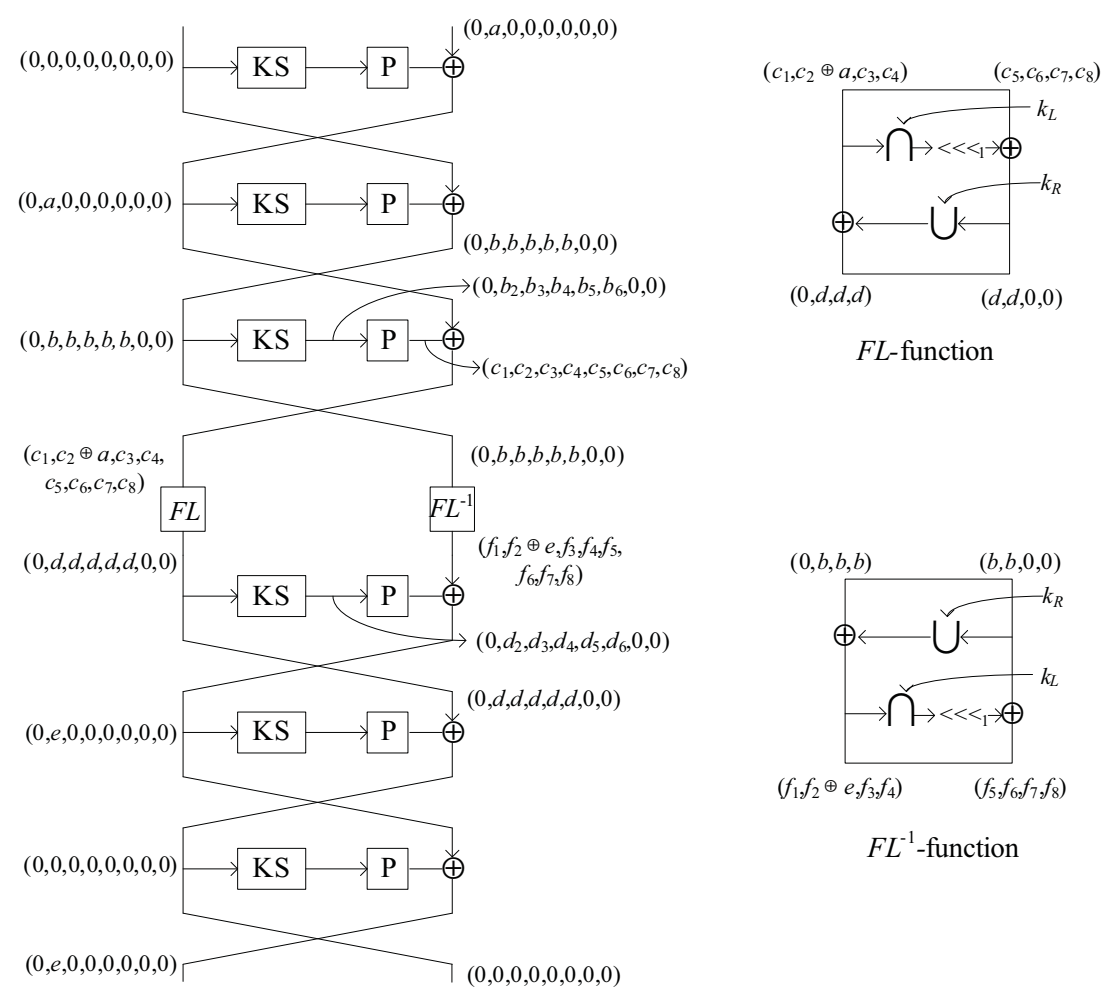

Fig. 4. 6-round impossible differential path with the $F L / F L^{-1}$ layer in the middle

where $a$ is arbitrary non-zero byte. The output difference of the first round is

$$
((0, a, 0,0,0,0,0,0) ;(0,0,0,0,0,0,0,0)) .
$$

Then by Property 1, the output differences of the second and third round are

$$
((0, b, b, b, b, b, 0,0) ;(0, a, 0,0,0,0,0,0)) \text { and }\left(\left(c_{1}, c_{2} \oplus a, c_{3}, c_{4}, c_{5}, c_{6}, c_{7}, c_{8}\right) ;(0, b, b, b, b, b, 0,0)\right)
$$

with probability 1 , as long as

$$
\left(c_{1}, c_{2}, c_{3}, c_{4}, c_{5}, c_{6}, c_{7}, c_{8}\right)=P\left(0, b_{2}, b_{3}, b_{4}, b_{5}, b_{6}, 0,0\right),
$$

where $b, b_{2}, b_{3}, b_{4}, b_{5}, b_{6}$ are unknown non-zero bytes, $\left(0, b_{2}, b_{3}, b_{4}, b_{5}, b_{6}, 0,0\right)$ is evolved from $(0, b, b, b, b, b, 0,0)$ after the S-box layer and $c_{l}(l=1, . ., 8)$ are unknown bytes.

Similarly, in the backward direction, we know that for arbitrary non-zero byte $e$, if the output difference of the sixth round is

$$
((0, e, 0,0,0,0,0,0) ;(0,0,0,0,0,0,0,0))
$$

then the input difference of the forth round is

$$
\left((0, d, d, d, d, d, 0,0) ;\left(f_{1}, f_{2} \oplus e, f_{3}, f_{4}, f_{5}, f_{6}, f_{7}, f_{8}\right)\right),
$$

where $d$ is an unknown non-zero byte and $f_{l}(l=1, . ., 8)$ are unknown bytes.

Now the input and output differences of the $F L$ function are determined. It can be deduced from Property 4 that $c_{3}=d$ and $c_{4}=d$, which means $c_{3}=c_{4}$. But this implies $b_{4}=0$ as

$$
\begin{aligned}
& c_{3}=b_{2} \oplus b_{3} \oplus b_{5} \oplus b_{6}, \\
& c_{4}=b_{2} \oplus b_{3} \oplus b_{4} \oplus b_{5} \oplus b_{6},
\end{aligned}
$$

which contradict $b_{4} \neq 0$. (By the input and output difference of $F L^{-1}$ function, we can also deduce another contradiction that $\left.d_{4}=0 \nLeftarrow d_{4} \neq 0\right)$. As a result, the differential 

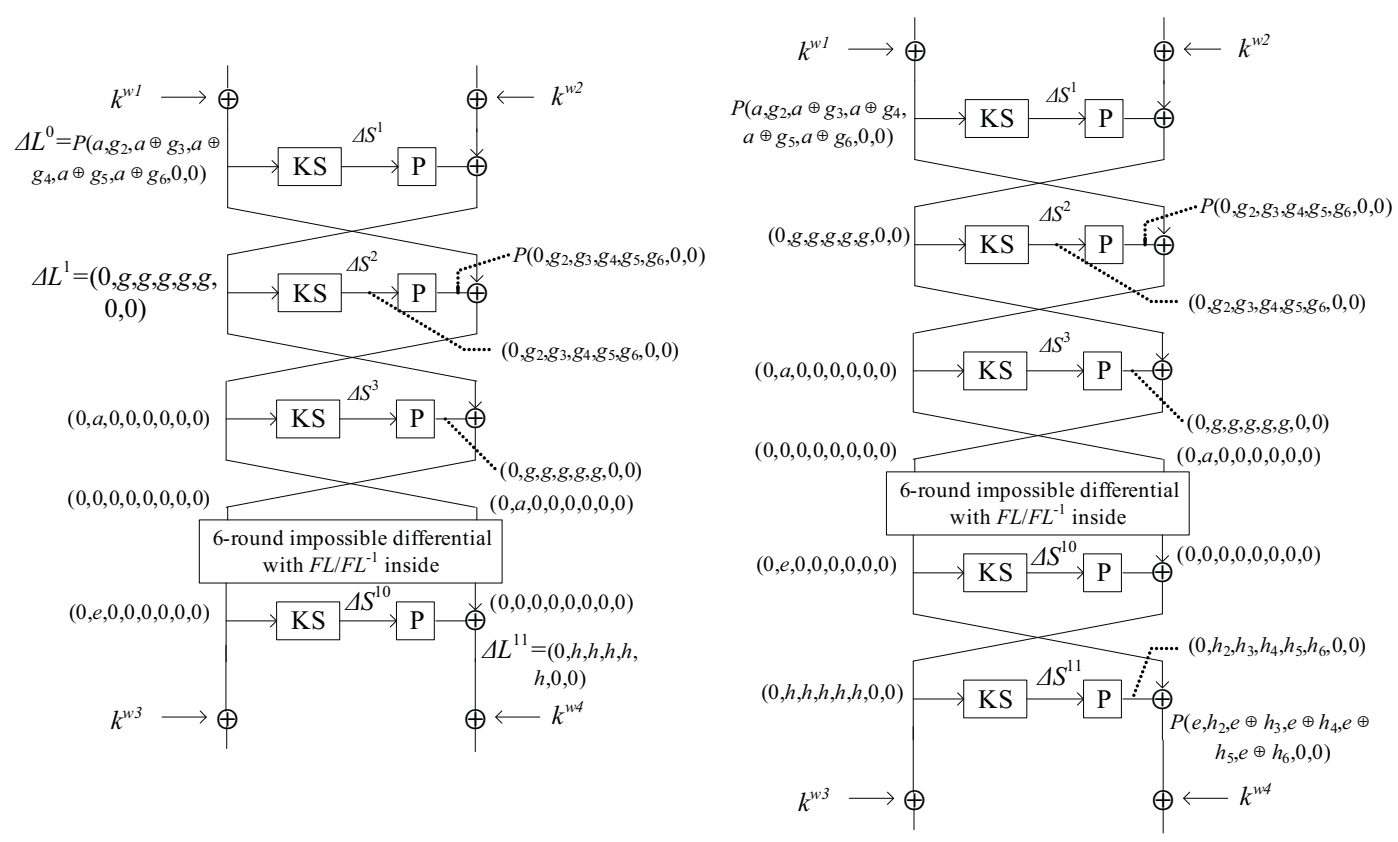

Fig. 5. Impossible Differential Attacks on 10-round Camellia-192 and 11-round Camellia-256 with whitening and $F L / F L^{-1}$

$$
((0,0,0,0,0,0,0,0) ;(0, a, 0,0,0,0,0,0)) \stackrel{6-\text { round }}{\longrightarrow}((0, e, 0,0,0,0,0,0) ;(0,0,0,0,0,0,0,0))
$$

is impossible.

Actually, there are three more 6-round impossible differential paths with the $F L / F L^{-1}$ layer in the middle, which are:

$$
\begin{aligned}
& ((0,0,0,0,0,0,0,0) ;(a, 0,0,0,0,0,0,0)) \underset{f-r o u n d}{\nrightarrow}((e, 0,0,0,0,0,0,0) ;(0,0,0,0,0,0,0,0)) \\
& ((0,0,0,0,0,0,0,0) ;(0,0, a, 0,0,0,0,0)) \stackrel{6-r o u n d}{\nrightarrow}((0,0, e, 0,0,0,0,0) ;(0,0,0,0,0,0,0,0)) \\
& ((0,0,0,0,0,0,0,0) ;(0,0,0, a, 0,0,0,0)) \stackrel{6-r o u n d}{\nrightarrow}((0,0,0, e, 0,0,0,0) ;(0,0,0,0,0,0,0,0))
\end{aligned}
$$

\section{Impossible Differential Attack on Camellia with $F L / F L^{-1}$ functions and whitening}

In this section, we present impossible differential attacks on 11-round Camellia-256 and 10-round Camellia-192 using the impossible differential in Section 3 .

\subsection{Impossible Differential Attack on 11-Round Camellia-256}

We add 3 rounds on the top and 2 rounds on the bottom of the 6-round impossible differential path to analysis 11-round Camellia-256, see Fig. 5 in the right. Denote $k^{a}=k^{w 1} \oplus k^{1}, k^{b}=$ $k^{w 2} \oplus k^{2}, k^{c}=k^{w 1} \oplus k^{3}, k^{d}=k^{w 4} \oplus k^{10}$ and $k^{e}=k^{w 3} \oplus k^{11}$. The attack is started by carrying out a precomputation.

Precomputation. A precomputational table $H$ for Rounds 2-3 is set up here, which contains the all possible pairs that can follow the differential in Rounds 2-3 and their corresponding subkeys $k^{b}, k_{2}^{c}$. This table can also be used for Rounds 10-11, as in the backward direction, the differences are the same as that of Rounds 2-3. The table is constructed as follows: 
For every $\left(L^{1}, g, k^{b}, L_{2}^{2}, a, k_{2}^{c}\right)$, compute $L^{\prime 1}=L^{1} \oplus(0, g, g, g, g, g, 0,0), T=F\left(L^{1}, k^{b}\right), T^{\prime}=$ $F\left(L^{\prime 1}, k^{b}\right), \Delta T=T \oplus T^{\prime}$ and sieve the ones satisfying $S\left(L_{2}^{2} \oplus k_{2}^{c}\right) \oplus S\left(L_{2}^{2} \oplus a \oplus k_{2}^{c}\right)=g$, where $g$ and $a$ are non-zero bytes. There are $2^{160}\left(L^{1}, g, k^{b}, L_{2}^{2}, a, k_{2}^{c}\right)$, and $2^{152}$ of which remain after the sieve. Then insert $\left(k^{b}, k_{2}^{c}\right)$ into the row indexed by $\left(L^{1}, g, \Delta T \oplus a, L_{2}^{2} \oplus T_{2}\right)$. Because there are only $2^{40} \Delta T$ which lead to $2^{48} \Delta T \oplus a$, there are $2^{128}$ rows in $H$ and each row contains $2^{24}$ 72 -bit subkeys $\left(k^{b}, k_{2}^{c}\right)$. Consequently, the memory complexity of the table is about $2^{155.2}$ bytes and the time complexity of the precomputation is less than $2^{161}$ one round encryptions.

Data Collection. Choose $2^{n}$ structures of plaintexts, and each structure contains plaintexts with the following form:

$$
\left(P\left(y_{1}, y_{2}, y_{3}, y_{4}, y_{5}, y_{6}, \alpha, \beta\right) ;\left(x_{1}, x_{2}, x_{3}, x_{4}, x_{5}, x_{6}, x_{7}, x_{8}\right)\right)
$$

where $y_{i}(i=1, \ldots, 6)$ and $x_{j}(j=1, \ldots, 8)$ take all possible values and $\alpha, \beta$ are fixed in each structure. As a result, there are $2^{112}$ plaintexts in each structure and we can get $2^{n} \times 2^{112 \times 2-1}=$ $2^{n+223}$ plaintext pairs totally. For each of the pairs, $\Delta\left(P^{-1}\left(L^{0}\right)\right)_{7}=0, \Delta\left(P^{-1}\left(L^{0}\right)\right)_{8}=0$.

Ask for the encryptions of the plaintexts in each structure to get the corresponding ciphertexts, and keep the pairs whose ciphertext differences satisfy the following form by birthday attack:

$$
\left((0, h, h, h, h, h, 0,0) ; P\left(e, h_{2}, e \oplus h_{3}, e \oplus h_{4}, e \oplus h_{5}, e \oplus h_{6}, 0,0\right)\right),
$$

where $e, h, h_{2}, h_{3}, h_{4}, h_{5}$ and $h_{6}$ are non-zero bytes. So there are $2^{n+223-72}=2^{n+151}$ pairs remaining.

Key Recovery. In the key recovery procedure, we use Property 2 and the precomputational table to discard the wrong keys.

1. Individually guess $k_{l}^{a}(l=1,3, \ldots, 8)$ and check whether the equation $\triangle S_{l}^{1}=\left(P^{-1}\left(\triangle R^{0}\right)\right)_{l}$ holds. About $2^{n+151} \times 2^{-56}=2^{n+95}$ pairs will be kept. Next guess $k_{2}^{a}$, so $\left(L^{1}, L^{\prime 1}\right)$ can be computed. For each of the remaining pairs, do Step 2.

2. Initialize a table $\Gamma$ of $2^{144}$ all possible values $\left(k^{b}, k_{2}^{c}, k^{e}, k_{2}^{d}\right)$, for each of the remaining pairs, access the row $\left(L^{1}, \Delta L_{2}^{1}, \Delta R^{1}, R_{2}^{1}\right)$ and the row $\left(L^{11}, \Delta L_{2}^{11}, \Delta L^{12}, L_{2}^{12}\right)$ in table $H$. Then combining the values in the two rows to get $\left(k^{b}, k_{2}^{c}, k^{e}, k_{2}^{d}\right)$, and remove the corresponding value from $\Gamma$.

3. If $\Gamma$ is not empty, output the 208-bit value $\left(k^{a}, k^{b}, k_{2}^{c}, k_{2}^{d}, k^{e}\right)$, otherwise go to Step 1 and try another guess. The main key can be recovered when $\left(k^{a}, k^{b}, k_{2}^{c}, k_{2}^{d}, k^{e}\right)$ is obtained, which will be described as follows.

The following equations are deduced from Table 3 in [1]:

$$
\begin{aligned}
k^{a} & =\left(K_{L} \lll 0\right)_{L} \oplus\left(K_{B} \lll 0\right)_{L}, \\
k^{b} & =\left(K_{L} \lll 0\right)_{R} \oplus\left(K_{B} \lll 0\right)_{R}, \\
k^{c} & =\left(K_{L} \lll 0\right)_{L} \oplus\left(K_{R} \lll 15\right)_{L}, \\
k^{e} & =\left(K_{B} \lll 111\right)_{L} \oplus\left(K_{A} \lll 45\right)_{L}, \\
k^{d} & =\left(K_{B} \lll 111\right)_{R} \oplus\left(K_{L} \lll 45\right)_{R} .
\end{aligned}
$$

We guess every possible value of $K_{L}$, for each guess, $K_{B}$ can be calculated by Equations (2) and (3), then sieve this $\left(K_{L}, K_{B}\right)$ pair by Equation (6). For the $\left(K_{L}, K_{B}\right)$ that satisfy Equation (6), further compute 64 bits of $K_{A}$ by Equation (5). Then guess the other 64-bits of $K_{A}$, by the key schedule of Camellia-256, $K_{R}$ can be fully determined by $K_{B}$ and $K_{A}$. Equation (4) will further reduce the keys by a factor of $2^{8}$. So we get about $2^{192} \times 2^{-8} \times 2^{-8}=$ $2^{176}\left(K_{L}, K_{R}\right)$ and the correct $K=K_{L}|| K_{R}$ can be obtained by trial encryption. 
Complexity. We choose $n=9$, then the data complexity is $2^{121}$ chosen plaintexts. Step 2 removes $2^{48}$ wrong $\left(k^{b}, k_{2}^{c}, k^{e}, k_{2}^{d}\right)$, for each pair remained after Step $1, \frac{2^{48}}{2^{144}}=2^{-96}$ of wrong $\left(k^{b}, k_{2}^{c}, k_{2}^{d}, k^{e}\right)$ are removed. Consequently, the number of remaining wrong 208-bit value $\left(k^{a}, k^{b}, k_{2}^{c}\right.$, $\left.k_{2}^{d}, k^{e}\right)$ after analyzing all the pairs is $2^{64} \times 2^{144} \times\left(1-2^{-96}\right)^{2^{104}} \approx 0$.

The complexity of Step 1 is about $2 \times\left(\sum_{i=1}^{7} 2^{128-8(i-1)} \times 2^{8 i}\right) \times \frac{1}{8}+2 \times 2^{64} \times 2^{104} \approx 2^{169}$ one round encryptions, equivalent to $2^{165.7}$ encryptions. There are $2^{24}$ values in $H$, so in Step $2,2 \times 2^{24}$ memory access to $H$ and $2^{48}$ memory access to $\Gamma$ are needed for each pair, which result in $2^{64} \times 2^{104} \times 2^{48}=2^{216}$ memory access, equivalent to $2^{216} \times \frac{1}{52} \times \frac{1}{11} \approx 2^{206.8}$ 11-round encryptions. The complexity of Step 3 is about $2^{184} 6$-round encryptions, so the time complexity is dominated by Step 2, which about $2^{206.8}$ encryptions. And the memory complexity is about $2^{155.2}$ bytes.

\subsection{Impossible Differential Attack on 10-Round Camellia-192}

We remove a round from the bottom of the 11-round attack, and give an attack on 10-round Camellia-192, see Fig. 5 in the left. The choice of plaintexts is the same as the 11-round attack, and the ciphertext pairs are sieved by the difference:

$$
(0, e, 0,0,0,0,0,0 ; 0, h, h, h, h, h, 0,0),
$$

where $e$ and $h$ are non-zero values. After the sieve, about $2^{120}$ pairs remain.

Denote the equivalent subkeys $k^{a}=k^{w 1} \oplus k^{1}, k^{b}=k^{w 2} \oplus k^{2}, k^{c}=k^{w 1} \oplus k^{3}$ and $k^{d}=k^{w 3} \oplus k^{10}$ The key recovery phase is as follows:

1. Guess $k_{2}^{d}$ and check whether $\Delta S_{2}^{10}=\Delta L_{2}^{11}$, the number of remaining pairs after this step is about $2^{112}$.

2. Individually guess $k_{l}^{a}(l=1,3, \ldots, 8)$ and check whether the equation $\triangle S_{l}^{1}=\left(P^{-1}\left(\triangle R^{0}\right)\right)_{l}$ holds. About $2^{112} \times 2^{-56}=2^{56}$ pairs will be kept. Next guess $k_{2}^{a}$, so $\left(L^{1}, L^{\prime 1}\right)$ can be computed. For each of the remaining pairs, do Step 3.

3. Initialize a table $\Gamma^{\prime}$ of $2^{72}$ all possible values $\left(k^{b}, k_{2}^{c}\right)$, for each of the remaining pairs, access the row $\left(L^{1}, \Delta L_{2}^{1}, \Delta R^{1}, R_{2}^{1}\right)$ in table $H$. For each value in the row, remove the corresponding value from $\Gamma^{\prime}$.

4. If $\Gamma^{\prime}$ is not empty, output the 144 -bit value $\left(k^{a}, k^{b}, k_{2}^{c}, k_{2}^{d}\right)$, otherwise try another guess. The main key can be recovered when $\left(k^{a}, k^{b}, k_{2}^{c}, k_{2}^{d}\right)$ is obtained by the similar method of the 11-round attack, except that there are only for equations that can be used:

$$
\begin{aligned}
k^{a} & =\left(K_{L} \lll 0\right)_{L} \oplus\left(K_{B} \lll 0\right)_{L}, \\
k^{b} & =\left(K_{L} \lll 0\right)_{R} \oplus\left(K_{B} \lll 0\right)_{R}, \\
k^{c} & =\left(K_{L} \lll 0\right)_{L} \oplus\left(K_{R} \lll 15\right)_{L}, \\
k^{d} & =\left(K_{B} \lll 111\right)_{L} \oplus\left(K_{L} \lll 45\right)_{R} .
\end{aligned}
$$

Again, we guess every possible value of $K_{L}$, for each guess $K_{B}$ can be calculated by Equations (7) and (8), then sieve this $\left(K_{L}, K_{B}\right)$ pair by Equation (10). For the $\left(K_{L}, K_{B}\right)$ that satisfy Equation (10), compute 8 bits of $K_{R}$ by Equation (9), and further guess the rest unknown 56 bits of $K_{R}$. Furthermore, we test whether the $\left(K_{L}, K_{R}\right)$ can pass the key schedule of Camellia-192. About $2^{184} \times 2^{-8} \times 2^{-128}=2^{48}$ keys will remain, and the correct $K=K_{L} \| K_{R}$ can be obtained by trial encryption.

In this attack, the time complexity is dominated by Step 4, which is about $2^{176} 6$-round encryptions, equivalent to about $2^{175} 10$-round encryptions. The memory complexity is the same as the 11-round attack. If we did not take the pre-/post- whitening key into account, the complexity would be about $2^{144}$ encryptions. 


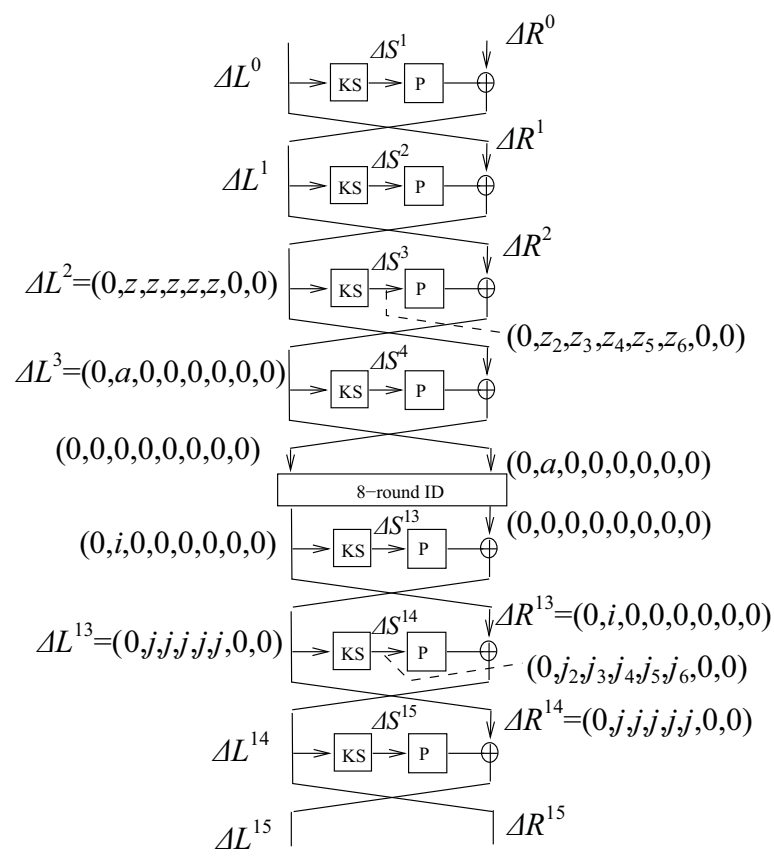

Fig. 6. Impossible Differential Attack on 15-round Camellia-256 without $F L / F L^{-1}$ layers and whitening

\section{Impossible Differential Cryptanalysis of 15-round Camellia-256 without $\boldsymbol{F L} / \boldsymbol{F} \boldsymbol{L}^{-1}$ layers and whitening}

In this section, we give an improved impossible differential attack on Camellia-256 by using a 8-round impossible differential path without $F L / F L^{-1}$ layer in Fig. 7, which was proposed in [18]. By adding 4 rounds on the top and 3 rounds on the bottom, we can attack 15-round Camellia-256 without $F L / F L^{-1}$ layers and whitening, see Fig. 6 .

Precomputation. First, we set up a table $\Gamma_{1}$ for the first two round of the 15 -round model. Property 2 implies that $\Delta L^{1}=P\left(a, z_{2}, z_{3} \oplus a, z_{4} \oplus a, z_{5} \oplus a, z_{6} \oplus a, 0,0\right)$, so we choose all the $2^{48}$ $\Delta L^{1}$ with the required form. Furthermore, all possible $\Delta L^{0}, L^{0} \oplus k^{1}$ and $\left(R^{0} \oplus k^{2}\right)_{2}$ are chosen. For each of the values, we compute $T=P\left(S\left(L^{0} \oplus k^{1}\right)\right), T^{\prime}=P\left(S\left(L^{0} \oplus k^{1} \oplus \Delta L^{0}\right)\right)$, and get the value $\left(R^{0} \oplus k^{2}\right)_{l}(l=1,3, \ldots, 8)$ by $\Delta L_{l}^{1},\left(P^{-1}\left(\Delta L^{0}\right)\right)_{l}, T$, and the corresponding differential table of S-box. Insert $L^{0} \oplus k^{1}$ and $R^{0} \oplus k^{2}$ into the row indexed by $\Delta R^{0}=T \oplus T^{\prime} \oplus \Delta L^{1}, \Delta L^{0}$, bits $61 \sim 64$ of $L^{0} \oplus k^{1}$, and bits $1 \sim 4,13 \sim 60$ of $R^{0} \oplus k^{2}$. As $2^{184}\left(\Delta L^{0}, \Delta L^{1}, L^{0} \oplus k^{1},\left(R^{0} \oplus k^{2}\right)_{2}\right)$ are totally chosen and there are $2^{184}$ rows in the table, we get about one $\left(L^{0} \oplus k^{1}, R^{0} \oplus k^{2}\right)$ in each row on average.

The complexity of the precomputation is about $2 \times 2^{184} 2$-round encryptions, equivalent to $2^{182.1} 15$-round encryptions. The table requires about $2^{184} \times 16=2^{188}$ bytes of memory.

Data Collection. For $2^{122.5}$ known plaintexts, ask for the encryptions and insert the ciphertexts into a hash table indexed by the 7 -th and 8 -th bytes of $P^{-1}\left(\Delta R^{15}\right)$. Since by Property 1 , the right half of ciphertexts must have the form

$$
\Delta R^{15}=\Delta L^{14}=P\left(i, j_{2}, j_{3} \oplus i, j_{4} \oplus i, j_{5} \oplus i, j_{6} \oplus i, 0,0\right) .
$$

By birthday attack, we can get $2^{244} \times 2^{-16}=2^{228}$ pairs that the 7 -th and 8 -th bytes of $P^{-1}\left(\Delta R^{15}\right)$ are 0 . 
Key Recovery. We give in Table 2 the corresponding positions of $k^{1}, k^{2}$ and $k^{15}$ in $K_{B}$, and the corresponding positions of $k^{3}, k^{14}, k_{2}^{4}$ and $k_{2}^{13}$ in $K_{R}$. It is obvious that there are close relations among the subkeys, i.e., there are common bits in some of the subkeys, which can be used to reduced the complexity of the attack. The key recovery phase is demonstrated as follows.

1. Individually guess $k_{l}^{15}(l=1,3, \ldots, 8)\left(K_{B}: 61 \sim 68,73 \sim 124\right)$ and remove the pairs do not satisfy $\Delta S_{l}^{15}=\left(P^{-1}\left(\Delta L^{15}\right)\right)_{l}$. About $2^{228-7 \times 8}=2^{172}$ pairs are kept. From Table 2 , bits $61 \sim 64$ of $k^{1}$ and bits $1 \sim 4,13 \sim 60$ of $k^{2}$ are known.

2. For each of the remaining pairs, compute bits $61 \sim 64$ of $L^{0} \oplus k^{1}$ and bits $1 \sim 4,13 \sim 60$ of $R^{0} \oplus k^{2}$, then access the value in the corresponding row in $\Gamma_{1}$. Insert $\left(\Delta L^{0}, \Delta R^{0}, L^{0}, R^{0}\right)$ to a table $\Gamma_{2}$ indexed by bits $1 \sim 60$ of $k^{1}\left(K_{B}: 1 \sim 60\right)$ and bits $5 \sim 12,61 \sim 64$ of $k^{2}$ $\left(K_{B}: 69 \sim 76,124 \sim 128\right)$. As a result, $\Gamma_{2}$ has $2^{72}$ rows with about $2^{100}\left(\Delta L^{0}, \Delta R^{0}, L^{0}, R^{0}\right)$ in each.

3. Guess bits $1 \sim 60$ of $k^{1}$ and bits $5 \sim 12,61 \sim 64$ of $k^{2}$, access the corresponding row in $\Gamma_{2}$, and compute $2^{100}\left(L^{2}, L^{\prime 2}\right)$ and $\left(R^{2}, R^{\prime 2}\right)$ by two-round encryptions as the whole $K_{B}$ is known.

4. From Property 1, it is clear that if a pair follows the path in Fig. 6, it has to satisfy $\Delta S_{l}^{14}=$ $\left(P^{-1}\left(\Delta L^{14}\right)\right)_{1} \oplus\left(P^{-1}\left(\Delta L^{14}\right)\right)_{l}(l=3, \ldots, 6)$ and $\Delta S_{2}^{14}=\left(P^{-1}\left(\Delta L^{14}\right)\right)_{2}$.

(a) Further guess $k_{2}^{14}\left(K_{R}: 5 \sim 12\right)$, partially decrypt round 15 and round 14 to discard the pairs which do not satisfy $\Delta S_{2}^{14}=\left(P^{-1}\left(\Delta L^{14}\right)\right)_{2}$. After this procedure, the number of remaining pairs is $2^{100-8}=2^{92}$.

(b) Individually guess $k_{l}^{14}(l=3, \ldots, 6)\left(k_{R}: 13 \sim 44\right)$ and keep the pairs which satisfy $\Delta S_{l}^{14}=\left(P^{-1}\left(\Delta L^{14}\right)\right)_{1} \oplus\left(P^{-1}\left(\Delta L^{14}\right)\right)_{l}$. There are $2^{92-8 \times 4}=2^{60}$ pairs being kept.

5. (a) Guess bits $45 \sim 47$ of $k_{R}$, now $k_{2}^{3}, k_{3}^{3}$, and $k_{4}^{3}\left(K_{R}: 24 \sim 47\right)$ are known. Detect if $\Delta S_{2}^{3}=\left(P^{-1}\left(\Delta R^{2}\right)\right)_{2}, \Delta S_{3}^{3}=\left(P^{-1}\left(\Delta R^{2}\right)\right)_{1} \oplus\left(P^{-1}\left(\Delta R^{2}\right)\right)_{3}$, and $\Delta S_{4}^{3}=\left(P^{-1}\left(\Delta R^{2}\right)\right)_{1} \oplus$ $\left(P^{-1}\left(\Delta R^{2}\right)\right)_{4}$. The number of remaining pairs is $2^{60-8 \times 3}=2^{36}$.

(b) Individually guess $k_{l}^{3}(l=5,6)\left(k_{R}: 48 \sim 63\right)$ and keep the pairs that satisfy $\Delta S_{l}^{3}=$ $\left(P^{-1}\left(\Delta R^{2}\right)\right)_{1} \oplus\left(P^{-1}\left(\Delta R^{2}\right)\right)_{l}$. There are $2^{36-8 \times 2}=2^{20}$ pairs being kept.

6. Guess $k_{1}^{14}\left(K_{R}: 125 \sim 128,1 \sim 4\right)$ (now the whole $k^{14}$ is known) and $k_{2}^{13}\left(K_{R}: 69 \sim 76\right.$ ), keep the pairs that meet $\Delta S_{2}^{13}=\Delta L_{2}^{13}$. The number of remaining of pairs will be $2^{20-8}=2^{12}$.

7. Guess the rest 8 bits of $k^{3}\left(K_{R}: 64 \sim 68,77 \sim 79\right)$, now the whole $k^{3}\left(K_{R}: 16 \sim 79\right)$ is known. We further guess $k_{2}^{4}\left(K_{R}: 88 \sim 95\right)$ and check if there is a pair satisfy $\Delta S_{2}^{4}=\Delta L_{2}^{2}$. If there is a pair satisfy this, then discard the key guess. Otherwise for every 219-bit key guess, exhaustively search the rest 37 bits of $K_{R}$ to calculate $K_{A}$, use the relation of $K_{A}$ and $K_{L}$ to recover $K_{L}$, and test the resulting $\left(K_{L}, K_{R}\right)$ by trial encryption.

Complexity. The data complexity is $2^{122.5}$ known plaintexts. In the data collecting phase, the computation of the 7 -th and 8 -th bytes of $P^{-1}\left(\Delta R^{16}\right)$ is less then $2 / 8$ one round encryption, so the complexity of computing the 7 -th and 8 -th bytes of $P^{-1}\left(\Delta R^{16}\right)$ is at most $2^{122.5} \times \frac{1}{4} \times \frac{1}{15} \approx$ $2^{116.6} 15$-round encryptions.

Below we elaborate the complexity of each step in the key-recovery phase.

1. The complexity is about $7 \times 2 \times 2^{8} \times 2^{228}=2^{240}$ one round encryptions, which is about $2^{236.1}$ encryptions.

2. This step needs about $2 \times 2^{56} \times 2^{172}=2^{229}$ memory access and $2^{72} \times 2^{100} \times 16=2^{176}$ bytes of memory.

3. The complexity of this step is about $2 \times 2^{128} \times 2^{100}=2^{229}$ two round encryptions.

4. (a) The complexity of this step is about $2 \times 2^{136} \times 2^{100}=2^{237}$ one round encryptions, which is about $2^{233.1}$ encryptions. 
(b) The complexity of the each operation in this step is about one round encryption, so the complexity of is about: $2 \times \sum_{i=0}^{3}\left(2^{144+8 \times i} \times 2^{92-8 \times i} \times \frac{1}{15}\right) \approx 2^{235.1}$.

5. (a) The complexity of this step is about $2 \times \frac{1}{15} \times 2^{171} \times\left(2^{60}+2^{52}+2^{44}\right) \approx 2^{228.1}$.

(b) The complexity of this step is about $2 \times \frac{1}{15} \times\left(2^{179} \times 2^{36}+2^{187} \times 2^{28}\right) \approx 2^{213.1}$.

6. This step requires $2 \times 2^{203} \times 2^{20} \times \frac{1}{15} \approx 2^{220.1}$ encryptions.

7. In step 8 , we expect $2^{219} \times\left(1-2^{-8}\right)^{2^{12}} \approx 2^{196.6}$ of the key guess remained. So about $2^{196.6+37}=2^{233.6}$ trail encryptions are request to recover the whole key. The complexity of this step is thus $2 \times 2^{219} \times\left[1+\left(1-2^{-8}\right)+\ldots+\left(1-2^{-8}\right)^{2^{12}}\right] \times \frac{1}{15}+2^{233.6} \approx 2^{233.6}$. As a result, the time complexity is dominated by Step 1 , which is about $2^{236.1} 15$-round encryptions.

\section{Conclusion}

In this paper, we present several 6-round impossible differential paths with $F L / F L^{-1}$ layers in the middle, which lead to impossible differential attacks on 10-round Camellia-192 and 11round Camellia-256. Then an impossible differential cryptanalysis of 15-round Camellia-256 without $F L / F L^{-1}$ layers and whitening is given by carefully using the subkey relation and a precomputational table. A summary of the previous attacks and our analysis of Camellia is given in Table 1.

Table 1. Summary of the attacks on Camellia

\begin{tabular}{ccccccc}
\hline Block Size & \#Rounds & $F L / F L^{-1}$ & Attack Type & Data & Time & Source \\
\hline Camellia-128 & 8 & $\times$ & Truncated DC & $2^{83.6} \mathrm{CP}$ & $2^{55.6}$ & {$[10]$} \\
& 9 & $\sqrt{ }$ & Square Attack & $2^{48} \mathrm{CP}$ & $2^{122}$ & {$[11$} \\
& 9 & $\times$ & Collision Attack & $2^{113.6} \mathrm{CP}$ & $2^{121}$ & {$[19]$} \\
& 9 & $\times$ & Square Attack & $2^{66} \mathrm{CP}$ & $2^{84.8}$ & {$[6]$} \\
\hline Camellia-192 & 11 & $\times$ & Impossible DC & $2^{118} \mathrm{CP}$ & $2^{126} \mathrm{MA}$ & {$[12$} \\
/-256 & 12 & $\times$ & Impossible DC & $2^{116.3} \mathrm{CP}$ & $2^{116.6}$ & {$[14]$} \\
& 10 & $\sqrt{ }$ & Square Attack & $2^{48} \mathrm{CP}$ & $2^{210}$ & {$[1]$} \\
& 11 rounds & $\sqrt{ }$ & Impossible DC & $2^{121} \mathrm{CP}$ & $2^{175}$ & this paper \\
& 11 & $\sqrt{ }$ & Imporssible DC & $2^{121} \mathrm{CP}$ & $2^{206.8}$ & this paper \\
& 12 & $\times$ & Impossible DC & $2^{120} \mathrm{CP}$ & $2^{181}$ & {$[18$} \\
& 12 & $\times$ & Linear Attack & $2^{119} \mathrm{KP}$ & $2^{247}$ & {$[16]$} \\
& 13 & $\times$ & Square Attack & $2^{66} \mathrm{CP}$ & $2^{249.6}$ & {$[6]$} \\
\hline & 15 & $\times$ & Impossible DC & $2^{120} \mathrm{CP}$ & $2^{211.7}$ & {$[12]$} \\
\hline
\end{tabular}

KP: known plaintext; CP: chosen plaintext; CC: chosen ciphertext; DC: differential attack

\section{References}

1. Aoki, K., Ichikawa, T., Kanda, M., Matsui, M., Moriai, S., Nakajima, J., Tokita, T.: Camellia: a 128-bit Block Cipher Suitable for Multiple Platforms-Design and Analysis. In: Stinson, D.R., Tavares, S. (eds.) SAC 2000. LNCS, vol. 2012, pp. 39-56. Springer, Heidelberg (2001)

2. Aoki, K., Ichikawa, T., Kanda, M., Matsui, M., Moriai, S., Nakajima, J., Tokita, T.: Specification of Camellia-a 128-bit Block Cipher. version 2.0 (2001), http://info.isl.ntt.co.jp/crypt/eng/camellia/specifications.html

3. Biham, E., Shamir, A.: Differential cryptanalysis of the Data Encryption Standard. Springer, Heidelberg (1993)

4. Biham, E., Biryukov, A., Shamir, A.: Cryptanalysis of Skipjack Reduced to 31 Rounds Using Impossible Differentials. In: Stern, J. (ed.) EUROCRYPT 1999. LNCS, vol. 1592, pp. 12-23. Springer, Heidelberg (1999)

5. CRYPTREC-Cryptography Research and Evaluation Committees, report, Archive (2002), http://www.ipa.go.jp/security/enc/CRYPTREC/index-e.html 
6. Duo, L., Li, C., Feng, K.: Square like attack on camellia. In: Qing, S., Imai, H., Wang, G. (eds.) ICICS 2007. LNCS, vol. 4861, pp. 269-283. Springer, Heidelberg (2007)

7. Hatano, Y., Sekine, H., Kaneko, T.: Higher order differential attack of Camellia (II). In: Nyberg, K., Heys, H.M. (eds.) SAC 2002. LNCS, vol. 2595, pp. 129-146. Springer, Heidelberg (2003)

8. International Standardization of Organization (ISO), International Standard- ISO/IEC 18033-3, Information technology-Security techniques-Encryption algorithms -Part 3: Block ciphers (2005)

9. Kühn, U.: Improved Cryptanalysis of MISTY1, In: Daemen, J. and Rijmen, V. (Eds.) FSE 2002, LNCS 2365, pp. 61-75. Springer-Verlag, Heidelberg (2002)

10. Lee, S., Hong, S.H., Lee, S.-J., Lim, J.-I., Yoon, S.H.: Truncated differential cryptanalysis of camellia. In: Kim, K.-c. (ed.) ICISC 2001. LNCS, vol. 2288, pp. 32-38. Springer, Heidelberg (2002)

11. Lei, D., Chao, L., Feng, K.: New observation on Camellia. In: Preneel, B., Tavares, S. (eds.) SAC 2005. LNCS, vol. 3897, pp. 51-64. Springer, Heidelberg (2006)

12. Lu, J.: Cryptanalysis of Block Ciphers. PhD Thesis, Department of Mathematics, Royal Holloway, University of London, England (2008)

13. Lu, J., Kim, J.-S., Keller, N., Dunkelman, O.: Improving the efficiency of impossible differential cryptanalysis of reduced camellia and MISTY1. In: Malkin, T.G. (ed.) CT-RSA 2008. LNCS, vol. 4964, pp. 370-386. Springer, Heidelberg (2008)

14. Mala, H., Shakiba, M., Dakhilalian, M., Bagherikaram G. New Results on Impossible Differential Cryptanalysis of Reduced-Round Camellia-128, SAC 2009, LNCS 5867, pp.281-294, 2009.

15. NESSIE-New European Schemes for Signatures, Integrity, and Encryption, final report of European project IST-1999-12324. Archive (1999), https://www.cosic.esat.kuleuven.be/nessie/Bookv015.pdf

16. Shirai, T.: Differential, Linear, Boomerang and Rectangle Cryptanalysis of Reduced-Round Camellia. In: Proceedings of the Third NESSIE Workshop, Munich, Germany, November 6-7 (2002)

17. Sugita, M., Kobara, K., Imai, H.: Security of Reduced Version of the Block Cipher Camellia against Truncated and Impossible Differential Cryptanalysis. In: Boyd, C. (ed.) ASIACRYPT 2001. LNCS, vol. 2248, pp. 193207. Springer, Heidelberg (2001)

18. Wu, W., Zhang, W., and Feng, D. Impossible Differential Cryptanalysis of Reduced-Round ARIA and Camellia. Journal of Computer Science and Technology, 22(3), 449-456.

19. Wenling, W., Dengguo, F., Hua, C.: Collision attack and pseudorandomness of reduced-round camellia. In: Handschuh, H., Hasan, M.A. (eds.) SAC 2004. LNCS, vol. 3357, pp. 252-266. Springer, Heidelberg (2004)

20. W. Wu, L. Zhang, and W. Zhang, Improved Impossible Differential Cryptanalysis of Reduced-Round Camellia. In: Avanzi, R., Keliher, L., Sica, F. (eds.) SAC 2008. LNCS, vol.5381, pp. 442-456. Springer, Heidelberg (2009) 


\section{A 8-Round Impossible Differential Path without $F L / F L^{-1}$ Layer}

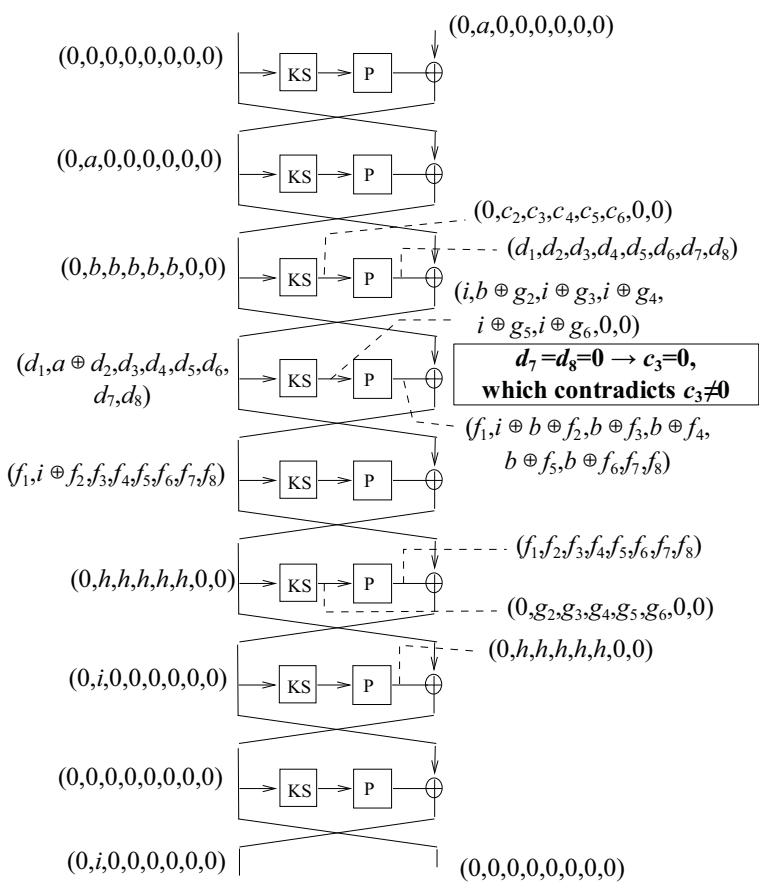

Fig. 7. 8-round Impossible Differential Path without $F L / F L^{-1}$ layer

\section{B The process of key recovery attack on 15-round Camellia-256 without} $\boldsymbol{F L} / \boldsymbol{F} L^{-1}$ layers and whitening

Table 2. Corresponding bit positions of the subkeys in $K_{B}$ and $K_{R}$

\begin{tabular}{c|c||c|c||c|c||c|c}
\hline $\begin{array}{c}\text { Subkey } \\
\text { bytes }\end{array}$ & $\begin{array}{c}\text { Bit positions } \\
\text { in } K_{B}\end{array}$ & $\begin{array}{c}\text { Subkey } \\
\text { bytes }\end{array}$ & $\begin{array}{c}\text { Bit positions } \\
\text { in } K_{B}\end{array}$ & $\begin{array}{c}\text { Subkey } \\
\text { bytes }\end{array}$ & $\begin{array}{c}\text { Bit positions } \\
\text { in } K_{R}\end{array}$ & $\begin{array}{c}\text { Subkey } \\
\text { bytes }\end{array}$ & $\begin{array}{c}\text { Bit positions } \\
\text { in } K_{R}\end{array}$ \\
\hline$k_{1}^{1}$ & $1 \sim 8$ & $k_{5}^{2}$ & $97 \sim 104$ & $k_{1}^{3}$ & $16 \sim 23$ & $k_{1}^{14}$ & $125 \sim 128,1 \sim 4$ \\
$k_{2}^{1}$ & $9 \sim 16$ & $k_{6}^{2}$ & $105 \sim 112$ & $k_{2}^{3}$ & $24 \sim 31$ & $k_{2}^{14}$ & $5 \sim 12$ \\
$k_{3}^{1}$ & $17 \sim 24$ & $k_{7}^{2}$ & $113 \sim 120$ & $k_{3}^{3}$ & $32 \sim 39$ & $k_{3}^{14}$ & $13 \sim 20$ \\
$k_{4}^{1}$ & $25 \sim 32$ & $k_{8}^{2}$ & $121 \sim 128$ & $k_{4}^{3}$ & $40 \sim 47$ & $k_{4}^{14}$ & $21 \sim 28$ \\
$k_{5}^{1}$ & $33 \sim 40$ & $k_{1}^{15}$ & $61 \sim 68$ & $k_{5}^{3}$ & $48 \sim 55$ & $k_{5}^{14}$ & $29 \sim 36$ \\
$k_{6}^{1}$ & $41 \sim 48$ & $k_{2}^{15}$ & $69 \sim 76$ & $k_{6}^{3}$ & $56 \sim 63$ & $k_{6}^{14}$ & $37 \sim 44$ \\
$k_{7}^{1}$ & $49 \sim 56$ & $k_{3}^{15}$ & $77 \sim 84$ & $k_{7}^{3}$ & $64 \sim 71$ & $k_{7}^{14}$ & $45 \sim 52$ \\
$k_{8}^{1}$ & $57 \sim 64$ & $k_{4}^{15}$ & $85 \sim 92$ & $k_{8}^{3}$ & $72 \sim 79$ & $k_{8}^{14}$ & $53 \sim 60$ \\
$k_{1}^{2}$ & $65 \sim 72$ & $k_{5}^{15}$ & $93 \sim 100$ & $k_{2}^{4}$ & $88 \sim 95$ & $k_{2}^{13}$ & $69 \sim 76$ \\
$k_{2}^{2}$ & $73 \sim 80$ & $k_{6}^{15}$ & $101 \sim 108$ & & & & \\
$k_{3}^{2}$ & $81 \sim 88$ & $k_{7}^{15}$ & $109 \sim 116$ & & & & \\
$k_{4}^{2}$ & $89 \sim 96$ & $k_{8}^{15}$ & $117 \sim 124$ & & & & \\
\hline
\end{tabular}

